\title{
Position statement on the use of Tenofovir Alafenamide for the treatment of chronic Hepatitis $B$ Virus infection in Africa
}

\begin{abstract}
The prevalence and morbidity of hepatitis B virus (HBV) infection is high in Africa. Scale-up of treatment with the use of nucleos(t)ide analogs (NAs) with high barrier to resistance such as entecavir (ETV), tenofovir disoproxil fumarate (TDF), or tenofovir alafenamide (TAF) may help curtail the disease burden in sub-Saharan Africa. However, limited accessibility to these NAs and limitations of ETV (development of resistance in lamivudine-exposed patients), and TDF (decline in renal function and bone mineral density with long-term use) act as barriers to optimization of HBV treatment in this region. Therefore, a panel of 9 (nine) hepatology experts from Africa convened, reviewed the literature, and developed the current position statement for use of TAF in resource-limited African settings. This article presents an overview of the efficacy and safety of TAF versus TDF in both HBeAgnegative and HBeAg-positive chronic HBV patients. Studies have revealed non-inferiority for the proportion of patients who had HBV DNA $<29 \mathrm{IU} / \mathrm{mL}$; higher rate of normalization of alanine aminotransferase levels; and better bone/renal safety, with TAF vs. TDF up to 144 weeks. Tenofovir alafenamide has better renal/bone safety compared to TDF, due to its less systemic exposure of tenofovir and greater exposure of tenofovir diphosphate to target cells. In view of these benefits of TAF, the expert panel proposed indications for scale-up of the use of TAF, specifically in patients with renal/bone health issues, in Africa. Optimization of use of TAF in the proposed patient population may help in lowering the prevalence and morbidity from HBV in Africa.
\end{abstract}

Keywords: hepatitis B virus, nucleoside analogs, tenofovir alafenamide, Africa
Volume II Issue 2 - 2020

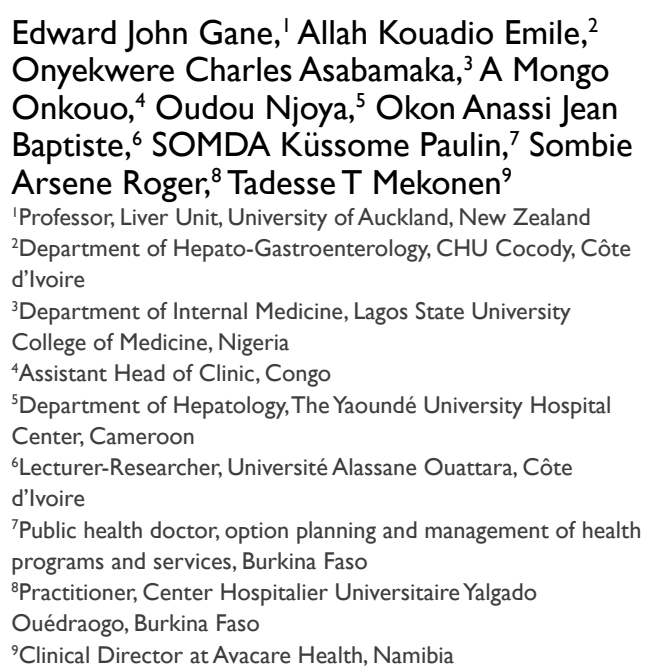

Correspondence: Prof Edward John Gane, Professor, Liver Unit, University of Auckland, Auckland, 1010, New Zealand, Tel +642I54837I, Email edgane@adhb.govt.nz

Received: February 06, 2020 | Published: March 04, 2020

\begin{abstract}
Abbreviations: HBV, hepatitis B virus; NAs, nucleos(t) ide analogs; TAF, tenofovir alafenamide; TDF, tenofovir disoproxil fumarate; ETV, entecavir; WHO, world health organization; $\mathrm{HBsAg}$, hepatitis B surface antigen; HBIG, hepatitis B immunoglobulin G; ALT, alanine aminotransferase; AASLD, american association for the study of liver diseases; ULN, upper limit of normal; LAM, lamivudine; YMDD, tyrosine-methionine-aspartate-aspartate
\end{abstract}

\section{Introduction}

According to the latest World Health Organization (WHO) estimates, globally, hepatitis B virus (HBV) infection was prevalent in about 257 million individuals (3.5\%) in 2015, with Africa having the second highest disease prevalence (60million HBV-infected individuals [6.1\%]). ${ }^{1}$ The Polaris Observatory Collaborators, in a modeling study that involved 120 countries, reported hepatitis B surface antigen (HBsAg) positivity to be $12.2,22.2,6.8$, and 39.1 million individuals in the Central, East, Southern, and West regions of subSaharan Africa, respectively. ${ }^{2}$ The reasons for the high prevalence of $\mathrm{HBV}$ infection in sub-Saharan Africa may be due to the high motherto-child transmission rate of the disease, which in the case of HBsAgpositive and hepatitis $\mathrm{B}$ e-antigen ( $\mathrm{HBeAg}$ )-positive mothers is about $40 \%$ in Africa. ${ }^{1}$ The high mother-to-child transmission rate of HBV infection in Africa may be due to the lack of access to antiviral therapy in pregnant women with high viral load, along with low uptake of birth dose vaccination and hepatitis B immunoglobulin G (HBIG) ${ }^{1,2}$
In 2015 , the birth dose vaccination coverage in Africa was only $10 \%{ }^{1}$ As a result of the low neonatal vaccination rates, the prevalence of HBsAg infection in children aged five years has been reported to be the highest in Africa (3.4\%; over a million HBsAg-positive children aged five years in 2016), which represents a significant barrier to achieving the WHO HBV elimination targets. ${ }^{2}$

In addition to the high prevalence, the morbidity from HBV infection is also high in Africa. Chronic HBV infection has been reported to be the leading cause of hepatocellular carcinoma (HCC) in several regions of Africa, ${ }^{3}$ and about $50 \%$ of liver cancer cases in subSaharan Africa have been noted to be caused by HBV infection. ${ }^{4}$ The mortality from chronic HBV infection is also high, both globally and in Africa. Globally, about 1.34 million deaths were attributed to viral hepatitis in 2015, of which $66 \%$ were due to chronic HBV infection, with Africa recording the third highest number of deaths $(136,000) .{ }^{1}$

Scale-up of treatment and care is therefore, crucial to help control the high prevalence, morbidity, and mortality due to HBV infection, both globally and in Africa. ${ }^{5}$

\section{Current guideline recommendations on treatment of chronic HBV infection}

The American Association for the Study of Liver Diseases (AASLD) 2018 guidelines recommend the nucleos(t)ide analogs 
(NAs), entecavir (ETV), tenofovir disoproxil fumarate (TDF), or tenofovir alafenamide (TAF) as the preferred first-line option for the treatment of adults with immune-active, chronic HBV infection, to decrease the risk of liver-associated complications. Tenofovir alafenamide or ETV is recommended as a preferable option in patients with or at risk of renal dysfunction or bone disease. In immunetolerant, chronic HBV-infected patients, the American guidelines recommend antiviral therapy only in select adults aged $>40$ years, with normal alanine aminotransferase (ALT) levels, but elevated HBV DNA $(1,000,000 \mathrm{IU} / \mathrm{mL})$ and presence of liver-associated complications such as necroinflammation or fibrosis. ${ }^{6}$

The European Association for the Study of the Liver (EASL) 2017 guidelines recommend NAs with a high barrier to HBV resistance, such as ETV, TDF, or TAF as the preferred first-line antiviral monotherapy in all adults with chronic HBV infection, with the below-mentioned recommendations for initiation of therapy:?

a. All HBeAg-positive or -negative chronic HBV-infected patients, defined by HBV DNA $>2000 \mathrm{IU} / \mathrm{mL}$, ALT greater than the upper limit of normal (ULN) and/or at least moderate liver necroinflammation or fibrosis, should be treated.

b. Patients with compensated or decompensated cirrhosis and detectable HBV DNA should be treated, regardless of ALT levels.

c. Treatment should be initiated in patients with HBV DNA $>20,000 \mathrm{IU} / \mathrm{mL}$ and ALT $>2 \times \mathrm{xLN}$, regardless of the degree of fibrosis.

d. Patients with $\mathrm{HBeAg}$-positive chronic HBV infection, defined by persistently normal ALT and high HBV DNA levels, may be treated if they are older than 30 years, regardless of the severity of liver histological lesions.

e. Patients with HBeAg-positive or -negative chronic HBV infection and family history of HCC or cirrhosis and extrahepatic manifestations can be treated, even if typical treatment indications are not fulfilled.

\section{Unmet needs in the use of Nucleos(t)ide analogs in africa}

Long-term NA treatment may be needed in most HBV-infected individuals in sub-Saharan Africa for complete suppression of HBV
DNA replication. ${ }^{5}$ However, accessibility issues may limit the use of NAs for the treatment of HBV monoinfection in sub-Saharan Africa. ${ }^{5,8}$ Further, there are several limitations to the use of ETV and TDF for the treatment of chronic HBV infection.

\section{Limitations of ETV}

Although ETV is a NA analog with a high barrier to HBV resistance, and only $1 \%-2 \%$ resistance reported in NA-naïve patients receiving long-term ETV monotherapy (over five years), ${ }^{9,10}$ the incidence of ETV resistance has been found to be high in patients with lamivudine (LAM) resistance. The probability of ETV resistance through 5 years of treatment in LAM-refractory patients has been found to be $43 \%$ and $51 \%$, with and without virological breakthrough, respectively. ${ }^{10}$ The resistance rate with ETV therapy has also been noted to be higher in LAM-exposed patients, with no detectable LAM resistance when compared to NA-naïve patients, thus indicating the need for monitoring during ETV monotherapy in LAM-exposed patients, regardless of the presence or absence of LAM resistance. ${ }^{9}$

Resistance to prolonged LAM monotherapy is due to the substitution of methionine in the tyrosine-methionine-aspartateaspartate (YMDD) motif at amino acid position 204 in the $\mathrm{C}$ domain of the HBV DNA polymerase enzyme gene, with valine or isoleucine. These changes are labeled as rtM204V/I, and are often associated with other compensatory mutations, such as rtL180M, rtV173L, and $\mathrm{rtL} 80 \mathrm{~V} / \mathrm{I}$, which enhance viral replication. ${ }^{11}$ Resistance to LAM increases with increased treatment duration and has been noted to be about $70 \%$ at five years of LAM therapy. ${ }^{12}$ Furthermore, LAM resistance may also be noted in chronic $\mathrm{HBV}$-infected patients with no history of antiviral therapy (NA-naïve patients or inactive $\mathrm{HBsAg}$ carriers), owing to spontaneous structural changes in the viral DNA polymerase enzyme. ${ }^{13,14}$ The pooled incidence of YMDD-motif mutations in LAM-untreated patients from eight countries across Asia and Europe has been found to be $12.21 \%{ }^{14}$ This finding, therefore, highlights the need for monitoring long-term ETV therapy, even in LAM-untreated or NA-naïve patients.

The use of a higher dose of ETV to prevent ETV resistance in patients with LAM-resistant HBV infection has been considered and evaluated in phase III studies with positive results. ${ }^{15}$ However, ETV has been found to be less effective than tenofovir in achieving rapid and complete virological suppression in LAM-exposed HBV patients. ${ }^{16}$

Table I Patients with HBV infection, suitable for treatment with TAF

\begin{tabular}{ll}
\hline Patients with HBV infection & Indications for TAF \\
\hline & Age $\geq 50$ years \\
At risk of bone and renal disease & Hemodialysis and CKD \\
& Postmenopausal women \\
& Obese patients (BMI $\left.>25 \mathrm{~kg} / \mathrm{m}^{2}\right)$ \\
& Use of chronic steroid therapy or medications that affect the bones \\
& History of fragility fracture \\
With bone disease & Osteoporosis \\
& eGFR $<60 \mathrm{~mL} / \mathrm{min} / \mathrm{I} .73 \mathrm{~m}^{2}$ \\
& Albuminuria $>30 \mathrm{mg}$ or moderate proteinuria \\
With renal abnormalities & Low phosphate levels $(<2.5 \mathrm{mg} / \mathrm{dL})$ \\
& Hemodialysis \\
& Impaired renal function \\
\hline
\end{tabular}


Table Continued...

\begin{tabular}{|c|c|}
\hline Patients with HBV infection & Indications for TAF \\
\hline \multirow{8}{*}{$\begin{array}{l}\text { With comorbidities at risk of renal } \\
\text { disease* }\end{array}$} & Hypertension \\
\hline & Type I and type 2 diabetes mellitus \\
\hline & Cardiovascular disease \\
\hline & Smoking \\
\hline & Patients at risk of vitamin D deficiency \\
\hline & Family history of kidney disease \\
\hline & Lupus \\
\hline & Multiple myeloma \\
\hline \multirow{6}{*}{$\begin{array}{l}\text { With comorbidities at risk of bone } \\
\text { disease* }\end{array}$} & History of fractures \\
\hline & Hyperparathyroidism \\
\hline & Nutritional or gastrointestinal problems (e.g., Crohn's or celiac disease) \\
\hline & $\begin{array}{l}\text { Hematological disorders or malignancy } \\
\text { Hypogonadal states (e.g. Turner syndrome/Klinefelter syndrome, } \\
\text { amenorrhea, etc.) }\end{array}$ \\
\hline & Endocrine disorders (e.g. Cushing's syndrome) \\
\hline & Immobility \\
\hline Taking drugs causing renal toxicity* & Anticoagulants, NSAIDs, calcineurin inhibitors \\
\hline Taking drugs causing bone toxicity* & $\begin{array}{l}\text { Chronic use of steroids, thyroid hormone treatment (L-thyroxine), } \\
\text { certain steroid hormones (medroxyprogesterone acetate, luteinizing } \\
\text { hormone-releasing hormone agonists), aromatase inhibitors, } \\
\text { antipsychotics, anticonvulsants, antiepileptic drugs, lithium, methotrexate, } \\
\text { antacids, and proton-pump inhibitors }\end{array}$ \\
\hline \multicolumn{2}{|c|}{ With cirrhosis, on dialysis, or following organ transplantation } \\
\hline
\end{tabular}

*The list is indicative and not all-inclusive. It is intended as a guidance/reference

HBV, hepatitis B virus; TAF, tenofovir alafenamide; CKD, chronic kidney disease; BMI, body mass index; GFR, glomerular filtration rate; NSAIDs, nonsteroidal anti-inflammatory drugs

\section{Limitations of TDF}

Tenofovir disoproxil fumarate is a prodrug of tenofovir, which undergoes hydrolysis and subsequent phosphorylation to its active metabolite, tenofovir diphosphate (TFV-DP), which in turn inhibits HBV reverse transcriptase activity, resulting in the termination of DNA chain. ${ }^{17}$ Although TDF has demonstrated potent antiviral activity in patients with chronic HBV infection with no resistance through eight years of use, ${ }^{18}$ its long-term use may result in: (1) decrease in estimated glomerular filtration rate and cumulative nephrotoxicity; and (2) reduction in bone mineral density (BMD) and increase in markers of bone turnover. ${ }^{19-23}$

\section{Rationale and methodology for development of position statement on TAF}

In view of the high prevalence, morbidity and mortality of HBV infection, and low rates of treatment with antiviral agents in Africa, along with limitations of ETV and TDF, a panel of 9 (nine) expert hepatologists from Africa convened, reviewed the literature, and developed position statements for TAF use in the resource-limited settings in Africa. The recommendations proposed in this article have been compiled from the clinical experience shared by experts along with current evidences from the literature.

\section{Tenofovir Alafenamide}

\section{Pharmacology}

Tenofovir alafenamide is a phosphonamidate prodrug of tenofovir, which enters the hepatocytes and is hydrolyzed to tenofovir, which is then metabolized intracellularly to the active metabolite, TFV-DP, a competitive inhibitor of HBV polymerase/reverse transcriptase (pol/ RT) that terminates the elongation of the viral DNA chain during the process of $\mathrm{HBV}^{24-26}$

The recommended dose of TAF is $25 \mathrm{mg}$ to be taken orally once daily with food. The Tmax of TAF is $0.48 \mathrm{~h}$, and the drug is highly bound to proteins $(80 \%)$. It is metabolized in the hepatocytes by carboxylesterase 1, and peripheral blood mononuclear cells by cathepsin A. The plasma half-life of TAF is $0.51 \mathrm{~h}$, and the drug is primarily eliminated in the feces $(37.1 \%)$, with less than $1 \%$ excreted through the kidneys. The systemic exposure of TAF is 1.9 -fold higher in subjects with severe renal impairment and 7.5\% lower in subjects with mild hepatic impairment, when compared to normal patients. However, no dosage adjustment is required in patients with estimated creatinine clearance $(\mathrm{CrCl}) \geq 15 \mathrm{~mL} / \mathrm{min}$, in patients with $\mathrm{CrCl}<15 \mathrm{~mL} / \mathrm{min}$ who are receiving hemodialysis or in those with hepatic impairment. There are no dosing recommendations for TAF in 
patients with $\mathrm{CrCl}<15 \mathrm{~mL} / \mathrm{min}$ who are not receiving hemodialysis. ${ }^{27,28}$ There is limited or no information on the use of TAF in pregnancy. Therefore, the use of TAF may be considered in pregnancy, only if necessary. There are no studies on the use of TAF in breast feeding although it is predicted that concentrations in breast milk would be extremely low. Current recommendation is that TAF should not be used during breast feeding. ${ }^{28}$

Tenofovir alafenamide is a substrate of P-glycoprotein (P-gp) and breast cancer resistance protein (BRCP). Drugs which are P-gp inducers (e.g., rifampicin, rifabutin, carbamazepine, phenobarbital or St. John's wort) may lower the plasma concentrations of TAF, leading to reduction in therapeutic activity. Further, co-administration of TAF with drugs that inhibit P-gp and BRCP may increase the plasma concentration of TAF. Therefore, administration of TAF with P-gp inducers or inhibitors or BRCP inhibitors is not recommended. ${ }^{27,28}$

Although both TAF and TDF are prodrugs of tenofovir and share the same intracellular active metabolite, TFV-DP, TAF has greater plasma stability, but rapidly converts to TFV inside cells. Hence, systemic exposure to tenofovir is $90 \%$ lower with TAF compared to TDF, whilst hepatocyte levels of TFV-DP are higher with TAF than TDF at therapeutic doses. ${ }^{29,30}$ This difference in metabolism explains the fewer safety concerns on renal function and bone health with TAF when compared to TDF.

\section{Clinical efficacy}

The efficacy of TAF has been evaluated in two large phase 3 clinical trials in $\mathrm{HBeAg}$-positive and $\mathrm{HBeAg}$-negative chronic $\mathrm{HBV}$ patients (study 0110 and study 0108 , respectively). Both the trials were randomized, double-blind, multinational, noninferiority studies, and enrolled patients aged $\geq 18$ years with chronic $\mathrm{HBV}$ infection with plasma $\mathrm{HBV}$ DNA $>20,000 \mathrm{IU} / \mathrm{mL}$, serum ALT $>60 \mathrm{U} / \mathrm{L}$ in men or $>38 \mathrm{U} / \mathrm{L}$ in women (and not more than ten times ULN), and estimated creatinine clearance of at least $50 \mathrm{~mL} / \mathrm{min}$. Both treatment-naïve and treatment-experienced patients were included in these studies. Patients in both studies were randomly assigned in a double-blind manner to receive TAF $25 \mathrm{mg}$ or TDF $300 \mathrm{mg}$ orally, once daily, in a 2:1 ratio, each with a matching placebo for up to 96 weeks. ${ }^{31,32}$ After the double-blind phase, while $50 \%$ of the enrolled patients had their double-blind phase extended for one additional year, the remaining patients received an open-label treatment with TAF $25 \mathrm{mg}$ orally once daily until week $144 .{ }^{33}$

The primary efficacy endpoint in both the studies was the proportion of patients who had HBV DNA $<29 \mathrm{IU} / \mathrm{mL}$ at week 48 in those who received at least one dose of the study drug. Some of the pre-specified efficacy endpoints included: (1) percentage of patients with ALT normalization at week 48 (defined as ALT above a ULN of $\leq 43 \mathrm{U} / \mathrm{L}$ for men and $\leq 34 \mathrm{U} / \mathrm{L}$ for women younger than 69 years of age; and $\leq 35 \mathrm{U} / \mathrm{L}$ for men and $\leq 32 \mathrm{U} / \mathrm{L}$ for women older than 69 years of age; and at no more than ten times the ULN by central laboratory normal range; or $30 \mathrm{U} / \mathrm{L}$ for men and 19U/L for women as per AASLD), and (2) proportion of patients with HBsAg loss and seroconversion to anti-HBs at week 48 . The key secondary safety endpoints included bone and renal safety. ${ }^{31,32}$

Study 110 was conducted at 161 centers across 19 countries. A total of 873 eligible HBeAg-positive chronic HBV patients were randomized to TAF $(n=581)$ or TDF $(n=292)$. At week 48, TAF was noninferior to TDF with respect to the primary endpoint; $64 \%$ of patients receiving TAF vs. $67 \%$ receiving TDF had HBV DNA $<29 \mathrm{IU} /$ $\mathrm{mL}(\mathrm{p}=0.25) .{ }^{31}$ These results were sustained through to week $96(73 \%$ receiving TAF vs. $75 \%$ receiving TDF reached the primary endpoint; $p=0.47) .{ }^{22}$ A significantly higher proportion of patients receiving TAF vs. TDF had normalized ALT levels, as per the AASLD criteria, at both week $48(45 \%$ vs. $36 \%$, respectively; $\mathrm{p}=0.014)$ and week 96 ( $52 \%$ vs. $42 \%$, respectively; $\mathrm{p}=0.003) .{ }^{22,31}$ A similar trend was noted as per the central laboratory criteria at week 96 (75\% vs. $68 \%$ in TAF vs. TDF groups had normalized ALT levels, respectively; $\mathrm{p}=0.017){ }^{22}$ Further, more patients in the TAF group (14\%) experienced $\mathrm{HBeAg}$ loss when compared to the TDF group (12\%) at week 48 , though the difference was not statistically significant. Similarly, $10 \%$ of patients treated with TAF achieved $\mathrm{HBeAg}$ seroconversion compared to $8 \%$ of patients treated with TDF at week 48, with no significant difference between both the groups. There was no significant difference between both the groups in terms of HBsAg loss or seroconversion at week $48 .{ }^{31}$

In study 108, a total of 426 eligible HBeAg-negative chronic HBV patients were randomized to TAF $(n=285)$ or TDF $(n=141)$ at 105 sites. The findings revealed that TAF was noninferior to TDF at week $48 ; 94 \%$ vs. $93 \%$ of patients on TAF vs. TDF reached the primary endpoint, respectively $(\mathrm{p}=0.47) .{ }^{32}$ The non-inferiority of TAF vs. TDF was sustained at week $96 ; 90 \%$ vs. $91 \%$ of patients on TAF vs. TDF reached the primary endpoint, respectively $(\mathrm{p}=0.84) .{ }^{22}$ A significantly higher percentage of patients on TAF vs. TDF had normalized ALT levels at week 96 (by central laboratory criteria: $81 \%$ vs. $71 \%$, respectively, $\mathrm{p}=0.038$; by AASLD criteria: $50 \%$ vs. $40 \%$, respectively, $\mathrm{p}=0.035$ ). Further, patients receiving TAF had higher rates of ALT normalization at every study visit after week 4 when compared to patients receiving TDF. ${ }^{22}$ There was no significant difference between the two groups in terms of $\mathrm{HBeAg}$ or HBsAg seroconversion or loss, both at weeks 48 and $96 .{ }^{22,32}$

About 1118 patients were included in the analysis of the results of the double-blind extension phase of studies 108 and $110(759 \mathrm{HBeAg}$ positive and $359 \mathrm{HBeAg}$-negative patients; 866 on TAF and 252 on TDF). The primary endpoint or virologic control achieved at 96 weeks was sustained at 144 weeks, with TAF vs. TDF (HBeAg-negative patients: TAF: $87 \%$ vs. TDF: $85 \%$; HBeAg-positive patients: TAF: $74 \%$ vs. TDF: $71 \%$ ). A significantly greater proportion of patients on TAF achieved ALT normalization at year 3 in both the studies (HBeAg-negative patients: TAF: $71 \%$ vs. TDF: $59 \%, \mathrm{p}=0.052$; HBeAg-positive patients: TAF: $64 \%$ vs. TDF: $53 \%$, $\mathrm{p}=0.010$ ). There was no significant difference between the two groups in terms of $\mathrm{HBeAg}$ or HBsAg loss. ${ }^{33}$

\section{Resistance}

Tenofovir alafenamide has demonstrated potent in vitro antiviral activity against LAM-, ETV-, and adefovir-resistant isolates when compared to the wild-type HBV clinical isolates. ${ }^{34}$ In the pooled analysis of studies 108 and 110 at 96weeks, 87 patients in the TAF group met the eligibility criteria for resistance testing. Overall, no resistant isolates were detected in the TAF group at week $96{ }^{22,35}$ About 49 patients qualified for phenotypic analysis at week 144, and no isolates showed reduced susceptibility to TAF. ${ }^{36}$

\section{Safety and tolerability}

Treatment with TAF was well tolerated in both studies 108 and 110. The most common adverse events were upper respiratory tract infection, headache, and nasopharyngitis..$^{31,32}$

Regarding bone safety, in study 108, the percentage of patients with decline in BMD was significantly lower in the TAF vs. TDF 
groups at week 48 (hip: $-0.29 \%$ vs. $-2.16 \%$, respectively [p $<0.0001]$; spine: $-0.88 \%$ vs. $-2.51 \%$, respectively $[\mathrm{p}<0.0001]) .^{32}$ Similar findings were noted in study 110 at week 48, wherein a significantly lesser percentage of patients treated with TAF experienced decline in BMD compared to those treated with TDF (hip: $-0.10 \%$ vs. $-1.72 \%$, respectively $[\mathrm{p}<0.0001]$; spine: $-0.42 \%$ vs. $-2.29 \%$, respectively $[\mathrm{p}<0.0001]) .{ }^{31}$ In both the studies, significantly fewer patients treated with TAF vs. TDF experienced greater than $3 \%$ reduction in BMD at the hip ( $8 \%$ vs. $24 \%$ in HBeAg-positive patients, and $10 \%$ vs. $33 \%$ in $\mathrm{HBeAg}$-negative patients, respectively) and spine (18\% vs. $38 \%$ in $\mathrm{HBeAg}$-positive patients, and $22 \%$ vs. $39 \%$ in $\mathrm{HBeAg}$-negative patients, respectively) at week $48 .^{31,32}$ Biomarkers associated with bone resorption and formation showed significantly smaller changes from baseline at weeks 48 and 96 in patients treated with TAF vs. TDF in both the studies. ${ }^{22,23,31,32}$ The reduced effects of TAF vs. TDF on BMD decline were sustained at 96 weeks, and the magnitude of the difference between the two groups was significantly greater at week 96 vs. that noted at week $\left.48(\mathrm{p}<0.001){ }^{22,23}\right]$ Further, the mean percentage decline in both hip and spine BMD was also significantly low in the TAF vs. TDF group at year 3 or week 144 of the double-blind phase in both the studies (overall: hip: $-0.4 \%$ vs. $-2.5 \%$, respectively $[\mathrm{p}<0.001]$; spine: $-0.5 \%$ vs. $-2.0 \%$, respectively $[\mathrm{p}<0.001]){ }^{33}$

Regarding renal safety, at week 48 , there was a significantly smaller reduction in estimated glomerular filtration rate (eGFR) in the TAF vs. TDF groups in both the studies (median change in estimated GFR: HBeAg-negative patients: $-1.8 \mathrm{~mL} / \mathrm{min}$ vs. $-4.8 \mathrm{~mL} /$ $\mathrm{min}$; $\mathrm{p}=0.004$; HBeAg-positive patients: $-0.6 \mathrm{~mL} / \mathrm{min}$ vs. $-5.4 \mathrm{~mL}$ min; $\mathrm{p}<0.0001$ ) ${ }^{31,32}$ The results were sustained at week 96 (overall: $-1.2 \mathrm{~mL} / \mathrm{min}$ vs. $-4.8 \mathrm{~mL} / \mathrm{min}$ in the TAF vs. TDF groups, respectively; $\mathrm{p}<0.001)^{22}$ and week 144 of the double-blind phase (overall: $-1.2 \mathrm{~mL} /$ min vs. $-6.0 \mathrm{~mL} / \mathrm{min}$ in the TAF vs. TDF groups, respectively; $\mathrm{p}$ $<0.001){ }^{33}$

\section{Efficacy and safety of switchover from TDF to TAF}

In a single-arm, prospective, nonrandomized, cross-over, pharmacokinetic study, switchover from TDF to TAF resulted in a significant $90 \%$ decrease in plasma tenofovir concentrations (TDF: $99.98 \pm 2.24 \mathrm{ng} / \mathrm{mL}$ vs. TAF: $10.2 \pm 1.6 \mathrm{ng} / \mathrm{mL} ; \mathrm{p}<0.001$ ), and 2.41 -fold increase in cell-associated TFV-DP concentration (TAF: $834.7 \pm 2.49$ vs. TDF: $346.85 \pm 3.75 \mathrm{fmol} / 106$ cells; $p=0.004$ )[37]. This explains the improved bone and renal health with TAF vs. TDF.

In the open-label extension phase of studies 108 and 110 , of the 540 patients who switched from TDF to TAF at week 96, $284(53 \%)$ had $\geq 1$ risk factor and $123(23 \%)$ patients had $\geq 2$ risk factors. ${ }^{38}$ The preliminary results of switchover from TDF to TAF at 12 and 24weeks revealed maintenance of viral suppression, with significantly higher normalization of ALT levels as the AASLD criteria and improved bone and renal parameters with TAF therapy. ${ }^{39,40}$ The one-year results of the open-label extension phase also revealed maintenance of viral suppression in chronic HBV patients switched from TDF to TAF at 96 weeks. There was a significant increase in the proportion of patients with normalization of ALT levels after 48weeks of switchover or at week 144 of TAF therapy, both as per the AASLD and central laboratory criteria. The median change in creatinine clearance from baseline decreased significantly, and there was an improvement in markers of tubular dysfunction after 48 weeks of switchover (week 144 of TAF therapy) (overall decrease in serum creatinine: $-0.018 \pm 0.064 ; \mathrm{p}=0.008)$. There was also a significant decline in all markers of bone turnover and improvement in hip and spine BMD after 48weeks of switchover (hip BMD: $+0.97 \pm 2.88$, $\mathrm{p}=0.002$; spine BMD: $+2.18 \pm 3.36 ; \mathrm{p}<0.001) .{ }^{38,41}$ Further, patients who switched to TAF were also found to have significantly improved bone and renal safety at week 144 in comparison to those who remained on TDF for an additional year in the double-blind phase. The median change in eGFR in the TDF 3-year double-blind group vs. patients with TDF to TAF switch over from week 96 to week 144 was $-0.9 \mathrm{~mL} / \mathrm{min}$ vs. $+4.2 \mathrm{~mL} / \mathrm{min}(\mathrm{p}<0.001)$. The corresponding changes in hip BMD were -0.02 vs. $+0.98(\mathrm{p}<0.001)$ and spine $\mathrm{BMD}$ were +0.26 vs. +2.04 $(\mathrm{p}<0.001)$, respectively. ${ }^{42}$

\section{Expert panel recommendations on indications for the use of TAF rather than TDF}

In both studies 108 and 110, some proportion of patients had risk factors for bone or renal disease (or TDF toxicity). ${ }^{38}$ However, the presence of these risk factors such as age $\geq 50$ years, female gender, baseline eGFR $<90 \mathrm{~mL} / \mathrm{min} / 1.73 \mathrm{~m}^{2}$, or history of osteoporotic fracture had no influence on the study results. ${ }^{23,38} \mathrm{~A}$ significantly lower percentage of patients experienced a BMD decline of $>3 \%$ in the TAF vs. TDF groups, independent of the nature and frequency of these risk factors. $^{43}$

The decision on the treatment choice for chronic HBV infection should balance the benefits (the durability of response based on the severity of liver disease, and patient preference) vs. the risks (side effects, resistance, and cost).$^{44}$ The reviewed literature findings clearly indicate a potential benefit of TAF over interferon-based therapy, in terms of a better safety profile and patient preference; over ETV, in terms of better efficacy and low resistance; and over TDF, in terms of better bone and renal safety. A recently published national hepatitis action plan for South Africa has reported a plausible 3\% drop in the incidence of $\mathrm{HBV}$ infection with the addition of tenofovir therapy in HBV-infected individuals. ${ }^{45}$ Addressing the cost and accessibility issues can help improve the scale-up of TAF therapy and improve outcomes in HBV-monoinfected patients in Africa. ${ }^{46,47}$

\section{Summary and future directives}

Chronic HBV infection is a serious public health concern in Africa due to the high disease burden, high risk of mother-to-child disease transmission, low birth dose vaccination rates, overall low neonatal vaccination coverage, and limited accessibility to effective antiviral therapies. Tenofovir alafenamide is an effective and safe NA therapy with a low risk of resistance and favorable long-term treatment outcomes. It is a safer alternative to TDF and has a low risk of resistance in comparison to ETV. In addition to improving the neonatal vaccination coverage, scale-up of TAF therapy in Africa would help lower the disease burden and achieve the WHO HBV elimination targets.

\section{Acknowledgments}

We would like to thank Mylan Pharmaceuticals ltd for financial support in execution of this project. We would also like to thank BioQuest Solutions Ltd for providing writing assistance.

\section{Conflicts of interest}

Author Edward John Gane: Member of Clinical Advisory Board for Gilead Sciences, AbbVie, Janssen, Arrowhead, Merck, VIR Biotechnology, Assembly Bio. Member of Speakers' Bureau for Gilead Sciences, AbbVie, Mylan Pharmaceuticals. Rests of the authors have no conflict of interest. 


\section{Funding}

The project is supported by Mylan Pharmaceuticals Ltd.

\section{References}

1. World Health Organization (WHO). Global Hepatitis Report 2017. Geneva: World Health Organization; 2017. p. 83.

2. Razavi-Shearer D, Gamkrelidze I, Nguyen MH, et al. Global prevalence, treatment, and prevention of hepatitis B virus infection in 2016: A modelling study. Lancet Gastroenterol Hepatol. 2018;3(6):383-403.

3. Yang JD, Mohamed EA, Aziz AO, et al. Characteristics, management, and outcomes of patients with hepatocellular carcinoma in Africa: A multicountry observational study from the Africa Liver Cancer Consortium. Lancet Gastroenterol Hepatol. 2017;2(2):103-111.

4. Maucort-Boulch D, de Martel C, Franceschi S, et al. Fraction and incidence of liver cancer attributable to hepatitis $\mathrm{B}$ and $\mathrm{C}$ viruses worldwide. Int J Cancer. 2018;142(12):2471-2477.

5. Spearman CW, Afihene M, Ally R, et al. Hepatitis B in sub-Saharan Africa: Strategies to achieve the 2030 elimination targets. Lancet Gastroenterol Hepatol. 2017;2(12):900-909.

6. Terrault NA, Lok ASF, McMahon BJ, et al. Update on prevention, diagnosis, and treatment of chronic hepatitis B: AASLD 2018 hepatitis B guidance. Hepatology. 2018;67(4):1560-1599.

7. European Association for the Study of the Liver. EASL 2017 Clinical Practice Guidelines on the management of hepatitis B virus infection. $J$ Hepatol. 2017;67(2):370-398.

8. Howell J, Ladep NG, Lemoine M, et al. Hepatitis B in sub-Saharan Africa. South Sudan Med J. 2014;7(3):59-61.

9. Lee JH, Cho Y, Lee DH, et al. Prior exposure to lamivudine increases entecavir resistance risk in chronic hepatitis B patients without detectable lamivudine resistance. Antimicrob Agents Chemother. 2014;58(3):17301737.

10. Tenney DJ, Rose RE, Baldick CJ, et al. Long-term monitoring shows hepatitis B virus resistance to entecavir in nucleoside-naïve patients is rare through 5 years of therapy. Hepatology. 2009;49(5):1503-1514.

11. Warner N, Locarnini S. Mechanisms of hepatitis B virus resistance development. Intervirology. 2014;57(3-4):218-224.

12. Wright TL. Clinical trial results and treatment resistance with lamivudine in hepatitis B. Semin Liver Dis. 2004;24(Suppl 1):31-36.

13. Yildiz O, Aygen B, Demirturk N, et al. Lamivudine resistance mutations in patients infected with hepatitis B virus genotype D. World J Gastroenterol. 2011;17(45):4987-4992.

14. Tan Y, Ding K, Su J, et al. The naturally occurring YMDD mutation among patients chronically infected HBV and untreated with lamivudine: A systematic review and meta-analysis. PLoS One. 2012;7(3):e32789.

15. Sherman M, Yurdaydin C, Sollano J, et al. Entecavir for Treatment of Lamivudine-Refractory, $\mathrm{HBeAg}$-Positive Chronic Hepatitis B. Gastroenterology. 2006;130(7):2039-2049.

16. Cho EJ, Lee JH, Cho Y, et al. Comparison of the efficacy of entecavir and tenofovir in nucleos(t)ide analogue experienced chronic hepatitis B patients. PLoS One. 2015;10(6): 0130392.

17. Tenofovir disoproxil fumarate prescribing information.

18. Liu Y, Corsa AC, Buti M, et al. No detectable resistance to tenofovir disoproxil fumarate in $\mathrm{HBeAg}+$ and $\mathrm{HBeAg}-$ patients with chronic hepatitis B after 8 years of treatment. J Viral Hepat. 2017;24(1):68-74.
19. Lim YS, Gwak GY, Choi J, et al. Monotherapy with tenofovir disoproxil fumarate for adefovir-resistant vs. entecavir-resistant chronic hepatitis B: A 5-year clinical trial. J Hepatol. 2019;71(1):35-44.

20. Ahn SH, Kim W, Jung YK, et al. Efficacy and safety of besifovir dipivoxil maleate compared with tenofovir disoproxil fumarate in treatment of chronic hepatitis B virus infection. Clin Gastroenterol Hepatol. 2019;17(9):1850-1859.e4.

21. Vasudevan A, Ardalan ZS, Ahmed N, et al. Long-term safety and efficacy of tenofovir disoproxil fumarate substitution for hepatitis B immunoglobulin following liver transplantation. JGH Open. 2018;2(6):288-294.

22. Agarwal K, Brunetto M, Seto WK, et al. 96 weeks treatment of tenofovir alafenamide vs. tenofovir disoproxil fumarate for hepatitis B virus infection. J Hepatol. 2018;68(4):672-681.

23. Seto WK, Asahina Y, Brown TT, et al. Improved bone safety of tenofovir alafenamide compared to tenofovir disoproxil fumarate over 2 years in patients with chronic HBV infection. Clin Gastroenterol Hepatol. 2018. pii: S1542-3565(18)30633-30665.

24. Buti M, Riveiro-Baricela M, Esteban R. Tenofovir alafenamide fumarate: A new tenofovir prodrug for the treatment of chronic hepatitis B infection. J Infect Dis. 2017;216(S8):S792-S796.

25. Abdul Basit S, Dawood A, Ryan J, et al. Tenofovir alafenamide for the treatment of chronic hepatitis B virus infection. Expert Rev Clin Pharmacol. 2017;10(7):707-716.

26. Byrne R, Carey I, Agarwal K. Tenofovir alafenamide in the treatment of chronic hepatitis B virus infection: Rationale and clinical trial evidence. Ther Adv Gastroenterol. 2018;11:1-12.

27. Tenofovir alafenamide prescribing information.

28. https://www.ema.europa.eu/en/documents/product-information/ vemlidy-epar-product-information_en.pdf

29. Agarwal K, Fung SK, Nguyen TT, et al. Twenty-eight day safety, antiviral activity, and pharmacokinetics of tenofovir alafenamide for treatment of chronic hepatitis B infection. J Hepatol. 2015;62(3):533-540.

30. Babusis D, Phan TK, Lee WA, et al. Mechanism for effective lymphoid cell and tissue loading following oral administration of nucleotide prodrug GS-7340. Mol Pharm. 2013;10(2):459-466.

31. Chan HL, Fung S, Seto WK, et al. Tenofovir alafenamide versus tenofovir disoproxil fumarate for the treatment of $\mathrm{HBeAg}$-positive chronic hepatitis $\mathrm{B}$ virus infection: A randomised, double-blind, phase 3, non-inferiority trial. Lancet Gastroenterol Hepatol. 2016;1(3):185-195.

32. Buti M, Gane E, Seto WK, et al. Tenofovir alafenamide versus tenofovir disoproxil fumarate for the treatment of patients with $\mathrm{HBeAg}$-negative chronic hepatitis B virus infection: A randomised, double-blind, phase 3, non-inferiority trial. Lancet Gastroenterol Hepatol. 2016;1(3):196-206.

33. Chan HL, Lim YS, Seto WK, et al. Three-year efficacy and safety of tenofovir alafenamide (TAF) compared to tenofovir disoproxil fumarate (TDF) in $\mathrm{HBeAg}$-negative and $\mathrm{HBeAg}$-positive patients with chronic hepatitis B. Hepatology. 2018;68(1 Suppl):227A.

34. Liu Y, Miller MD, Kitrinos KM. Tenofovir alafenamide demonstrates broad cross-genotype activity against wild-type HBV clinical isolates and maintains susceptibility to drug-resistant $\mathrm{HBV}$ isolates in vitro. Antiviral Res. 2017;139:25-31.

35. Cathcart AL, Chan HL, Bhardwaj N, et al. No resistance to tenofovir alafenamide detected through 96 weeks of treatment in patients with chronic hepatitis B. Antimicrob Agents Chemother. 2018;62(10).

36. Chan HL, Marcellin P, Pan CQ, et al. No resistance to tenofovir alafenamide detected through 144 weeks of treatment in patients with chronic hepatitis B. Hepatology. 2018;68(1 Suppl):231A. 
37. Podany AT, Bares SH, Havens J, et al. Plasma and intracellular pharmacokinetics of tenofovir in patients switched from tenofovir disoproxil fumarate to tenofovir alafenamide. AIDS. 2018;32(6):761-765.

38. Gane E, Seto WK, Janssen H, et al. Safety and efficacy at 1 year after switching from tenofovir disoproxil fumarate to tenofovir alafenamide in chronic HBV patients with risk factors for TDF use. $J$ Hepatol. 2018;68(1):S65-S104.

39. http://www.natap.org/2017/EASL/WedemeyerEASLLC2017ViralHepatitisGeneralSession4.pdf Fong TL, Lee BT, Tien A, et al. Improvement of bone mineral density and markers of proximal renal tubular function in chronic hepatitis B patients switched from tenofovir disoproxil fumarate to tenofovir alafenamide. $J$ Viral Hepat. 2019;26(5):561-567.

40. Pan CQ, Brunetto MR, Hui AJ, et al. Improved Bone and Renal Safety at 1 Year After Switching From Tenofovir Disoproxil Fumarate to Tenofovir Alafenamide: Results From 2 Phase 3 Studies in HBeAg-Positive and HBeAg-Negative Patients with Chronic Hepatitis B. Washington, DC: The Liver Meeting; 2017.
41. Seto WK, Buti M, Izumi N, et al. Bone and renal safety are improved in chronic HBV patients 1 year after switching to tenofovir alafenamide (TAF) from tenofovir disoproxil fumarate (TDF). Hepatology. 2018;68(1 Suppl):240A.

42. Seto KW, Asahina Y, Peng CY, et al. Reduced changes in bone mineral density in chronic HBV patients receiving TAF compared to TDF.

43. Lok AS. Personalized treatment of hepatitis B. Clin Mol Hepatol. 2015;21(1):1-6.

44. Hecht R, Hiebert L, Spearman WC, et al. The investment case for hepatitis $\mathrm{B}$ and $\mathrm{C}$ in South Africa: Adaptation and innovation in policyanalysis for disease program scale-up. Health Policy Plan. 2018;33(4):528-538.

45. Lemoine M, Eholié S, Lacombe K. Reducing the neglected burden of viral hepatitis in Africa: Strategies for a global approach. J Hepatol. 2015;62(2):469-476.

46. Locarnini S, Hatzakis A, Chen DS, et al. Strategies to control hepatitis B: Public policy, epidemiology, vaccine and drugs. J Hepatol. 2015;62(1 Suppl):S76-S86. 